\title{
Multiband Microstrip Patch Antenna for Microwave Applications
}

\author{
Syed Ahsan Ali ${ }^{1}$, Umair Rafique ${ }^{2}$, Umair Ahmad ${ }^{3}$, M. Arif Khan ${ }^{4}$ \\ ${ }_{1,2,3,4}$ Department of Electronic Engineering, Mohammad Ali Jinnah University, Pakistan
}

\begin{abstract}
In this paper, we present a fractal shape slot based patch antenna for multiband operations. The fractal shape slot is designed on the rectangular patch and the antenna is fed through a microstrip line. The proposed design and feeding technique allows the antenna to operate at multiple frequencies in the range of 1$20 \mathrm{GHz}$. It is demonstrated through simulation that the Return Loss $(R L)$ values occurred at $1.33 \mathrm{GHz}, 4.16$ $\mathrm{GHz}, 7 \mathrm{GHz}, 9.8 \mathrm{GHz}, 12.7 \mathrm{GHz}, 15.5 \mathrm{GHz}$ and $18.3 \mathrm{GHz}$, respectively, having VSWR $\leq 2$. It is also observed that the gain of proposed design is higher than the conventional patch antenna. Parametric study is also included to give an overview for the performance of proposed design.
\end{abstract}

Keywords: fractal shape, patch antenna, multiband operations, microstrip line, parametric study.

\section{Introduction}

Increasing progress in communication system increases the demand of compact, cost effective and easily fabricated antennas. So, this requirement of present time is full-filled by the invention of patch antenna [1]. They are light weight, affordable, easy to manufacture and can easily be used in hand-held devices. Microstrip patch antenna is a metallic plate mounted on a dielectric of any kind. Mostly, low dielectric constant and thicker material is used for the designing of patch antenna. The patch has different shapes like circular, rectangular, ring and elliptical, respectively. Another metallic plate is mounted at the bottom of dielectric which is known as ground plane. Ground plane provides sufficient reflections to the fringing fields which occurred due to the change in length of patch antenna. Microstrip patch antenna can be fed by various techniques which are probe feed, microstrip line feed, proximity coupled feed and aperture/slot-coupled feed. Among all the advantages, there are few drawbacks of using patch antenna which are low gain, narrow bandwidth, high ohmic losses and low efficiency. Researchers proposed many techniques to overcome these disadvantages, but there is always a trade-off between the performance and design.

Recently, multiband patch antennas are investigated because of coverage of many wireless communication services such as GSM, DCS, CDMA and PCS [2, 3]. Many conventional techniques such as the use of PIN diodes, switches and varactor diodes are used for multiband operation $[4,5 \& 6]$. But, these designs provide reconfigurable frequency operations with bi-state ON/OFF control. Also, the use of active components increases complexity in the design and their use are difficult to handle because it needs extra biasing network. In [7], multiband characteristics were achieved through a triangular patch which operates in the range of 1.9-2.1 GHz. In this case, chip capacitors are used instead of varactor diodes. The impedance of an antenna is also controlled through chip capacitors which is a moderate technique.

In [8], a patch antenna was designed with a U-slot and by using PIN diodes. The PIN diodes are used to switch the slots ON and OFF for different frequency bands. But, it provides tri-band response in the reported frequency range. Another patch antenna was designed by incorporating two slots on both sides of the patch which are controlled by two switches. The status of these two switches changes the resonant frequencies which affects the frequency bands. In [9], a microstrip patch antenna was presented for wireless applications which woks from $0.9 \mathrm{GHz}$ to $5.35 \mathrm{GHz}$. A multiband response was achieved by designing a modified ground plane. It has a unique structure of comb shape on both sides of the patch. This technique also helps to enhance the bandwidth and gain of an antenna. A wideband microstrip patch antenna which also allowed multiband properties by designing an array was presented in [10]. This technique gives better efficiency and gain, operating at different bands which are $2.4 \mathrm{GHz}, 5.5 \mathrm{GHz}$ and $9 \mathrm{GHz}$, respectively. According to the presented results, it includes two satellite communication bands which are $\mathrm{C}$ and $\mathrm{X}$-band. These bands are also widely used in cellular communication, WLAN and radar communication.

To reduce the effective permittivity of substrate, a technique of Photonic Band-Gap (PBG) structure was introduced in [11]. The proposed multiband patch antenna resonates at six frequency bands in the range of 1-5.35 GHz. These bands cover most of the commercial communication applications. The designing of PBG structure on ground plane disgrade the efficiency of an antenna. Multiple patches with a slot-coupled technique were also used to get multiband results [12]. Three patches were used and a slot was etched in the middle of patch to couple upper and lower patches. This design worked at different bands in frequency range 0.7-1.7 GHz. It covers GPS, PCS and GSM frequency bands.

In this paper, a fractal shaped slot based microstrip patch antenna is designed and presented for multiple frequency operations. The proposed antenna design is able to operate in the frequency range of 1-20 
$\mathrm{GHz}$ having seven operating resonant frequencies which cover most of the desired communication bands. In addition, a VSWR which is $\leq 2$ is achieved according to the $10 \mathrm{~dB}$ bandwidth criteria. Also, the gain of an antenna is not achieved according to the criteria for lower frequency bands, but the gain achieved for upper frequencies is much higher than the conventional patch antenna.

\section{Antenna Geometry And Design}

The design and geometry of proposed antenna and fractal shape slot is shown in Fig .1. The antenna geometry consists of two dielectric layers in which the rectangular patch is designed on the upper substrate. The rectangular patch consists of a fractal shape slot which allows the antenna to radiate at multiple frequencies. The word fractal means that recursively generated structure having different dimensions. The dielectric used for the design purpose is Rogers RT/Duroid 5870 having relative permittivity 2.33 and thickness $1.57 \mathrm{~mm}$, respectively. The rectangular patch dimensions can be calculated as:

$$
L=\frac{1}{2 f_{r} \sqrt{\varepsilon_{\text {eff }}} \sqrt{\mu_{o} \varepsilon_{o}}}-2 \Delta L
$$

where

$$
\varepsilon_{e f f}=\frac{\varepsilon_{r}+1}{2}+\frac{\varepsilon_{r}-1}{2}\left[1+12 \frac{h}{W}\right]^{\frac{-1}{2}}
$$

and

$$
\frac{\Delta L}{h}=0.421 \frac{\left(\varepsilon_{e f f}+0.3\right)\left(\frac{W}{h}+0.264\right)}{\left(\varepsilon_{e f f}-0.258\right)\left(\frac{W}{h}+0.8\right)}
$$

where

$$
W=\frac{v_{o}}{2 f_{r}} \sqrt{\frac{2}{\varepsilon_{r}+1}}
$$

Hence, $W$ is the width of the patch, $L$ is the length of the patch, $\varepsilon_{e f f}$ is the effective dielectric constant of the material, $v_{o}$ is the speed of light in a vacuum, $f_{r}$ is the target frequency, $\varepsilon_{r}$ is the dielectric constant of the substrate, $h$ is the thickness of the substrate and $\Delta L$ represents the extension in length caused by the fringing effect and by considering the dimension of the patch, it can conformably be ignored.

The ground plane is sandwiched between the two dielectric layers. This technique is taken from the slot-coupled feed method of microstrip patch antenna design, because the placement of ground plane between the dielectric layers minimizes interference between radiating element and feed. By using this technique, an effective antenna structure can be obtained and maximum efficiency can be achieved.

The above presented geometry is then designed by using Ansoft HFSS. The overall dimensions of the rectangular patch and fractal shape slot is taken to demonstrate the antenna performance. The overall dimensions of the slot are shown in Fig. 1 (b) and the values according to the dimensions are given in Table 1. 


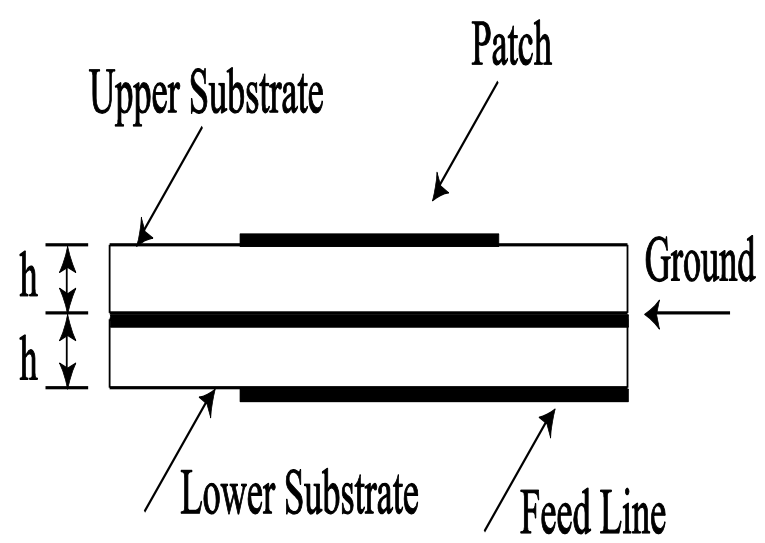

(a)

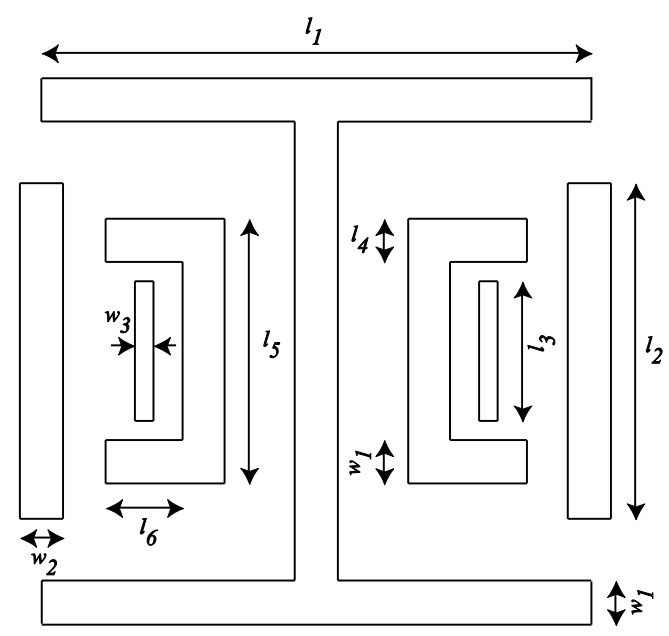

(b)

Fig. 1: (a) Geometry of proposed antenna and (b) dimensions of fractal shape slot within a patch.

Table 1. Design parameters of proposed patch antenna.
\begin{tabular}{|c|c|}
\hline Parameters & Values (mm) \\
\hline$l l$ & 20 \\
\hline$l 2$ & 14 \\
\hline$l 3$ & 4 \\
\hline$l 5$ & 2 \\
\hline$l 6$ & 9 \\
\hline 17 & 2 \\
\hline$w I$ & 20 \\
\hline$w 2$ & 2 \\
\hline$w 3$ & 1 \\
\hline$h$ & 1.57 \\
\hline
\end{tabular}

\section{Results And Discussion}

This section describes the simulation results of proposed multiband microstrip patch antenna. Discussion on the results is also provided in this section. Fig. 2 shows the simulated input return loss of proposed antenna. It is noticed that seven resonant frequencies occurred in the range of $1-20 \mathrm{GHz}$ which are $1.33 \mathrm{GHz}, 4.16 \mathrm{GHz}, 7 \mathrm{GHz}, 9.8 \mathrm{GHz}, 12.7 \mathrm{GHz}, 15.5 \mathrm{GHz}$ and $18.3 \mathrm{GHz}$, respectively. The resonant frequencies observed according to the $10 \mathrm{~dB}$ bandwidth criteria. The proposed fractal shape slot can be very useful to obtain a multiband response which is clear from Fig. 2. Also, VSWR noted at the resonant frequencies is $\leq 2$ which is the second major result of antenna design. The VSWR corresponding to the input return loss is shown in Fig. 3. One of the most and major design parameter is the impedance matching of an antenna. In the proposed design, the impedance matching is achieved by applying $50 \Omega$ input impedance to the microstrip line and by adjusting the width of feed line. A parametric study is obtained by changing the some slot dimensions and thickness of dielectric to demonstrate the performance of proposed design. Fig. 4 and 5 shows the simulated gain of proposed antenna. From Fig. 4, it is noticed that the proposed design is also compatible for receiving antenna system in the range of $1-10 \mathrm{GHz}$, because most of the frequency bands giving negative gain which is useful at receiver side. The maximum gain noted for lower frequency bands is $9 \mathrm{dBi}$ in the range of 0.8-1.4 $\mathrm{GHz}$, while minimum gain is $-7 \mathrm{dBi}$. Fig. 5 demonstrated that the proposed design is much suitable for satellite communication. As the frequency increases, gain is also increases. It can also be said that the gain is completely obeying the rule of input return loss for higher frequency bands. As the return loss approaches to zero, gain will increase with respect to it. The average gain noted for higher frequency bands is $5 \mathrm{dBi}$. 
Fig. 6 shows the simulated input return loss by changing the slot length $l_{l}$. When the length is adjusted lower than the actual length which is shown in Fig. 1 (a), there is no change observed in the return loss, but when the length is taken higher than the actual length, the impedance mismatch occurred at the most lower frequencies. There is not change observed at the higher frequencies. Also, the VSWR is also changed according to the return loss. Fig. 7 shows the simulated input return loss by changing the slot length $l_{2}$. When the length is adjusted lower than the actual length and all other parameters are kept constant, there is no effect in the return loss observed. But, when the length is chosen higher than the original value, there is a mismatch occurred between the input impedance and characteristics impedance of an antenna tends to maximum reflection to the input signal. This shows that the length $l_{2}$ plays a crucial role in the performance of proposed antenna design.

Fig. 8 shows the simulated input return loss by changing the slot length $l_{3}$. By adjusting the length lower and higher than the original value, there is no change noted in the result. This slot length provides complete impedance matching to antenna. One thing noticed that the minimum reflection occurred at most lower and higher frequencies, but this can be handled by optimizing the slot length according to the need. Fig. 9 shows the simulated input return loss results by changing the dielectric thickness $(h)$. In this case, the permittivity of the material is kept constant as given in Section II and the thickness is changed from lower to higher value. It is noticed, when minimum value is taken which is $1.27 \mathrm{~mm}$, antenna gives better response as compared to the other parametric study. Also, it is noticed that gradually increase in thickness will affect the antenna performance. So, from this observation it can be say that the use of thin substrate is also useful for the presented design.

\section{Conclusion}

A multiband fractal slot shaped microstrip patch antenna is designed for microwave applications. Seven resonant frequencies are achieved according to $10 \mathrm{~dB}$ bandwidth criteria. The resonant frequencies occurred in the range of 1-20 GHz. Also, the VSWR of proposed antenna is $\leq 2$. The maximum gain achieved for lower frequency bands is up to $9 \mathrm{dBi}$ and minimum gain is $-7 \mathrm{dBi}$ and the average gain achieved for the higher frequency bands is $5 \mathrm{dBi}$. Parametric study is also obtained to demonstrate the performance of proposed antenna. The proposed design is useful for Bluetooth ${ }^{\circledR}$, WLAN, radar and satellite communication, etc.

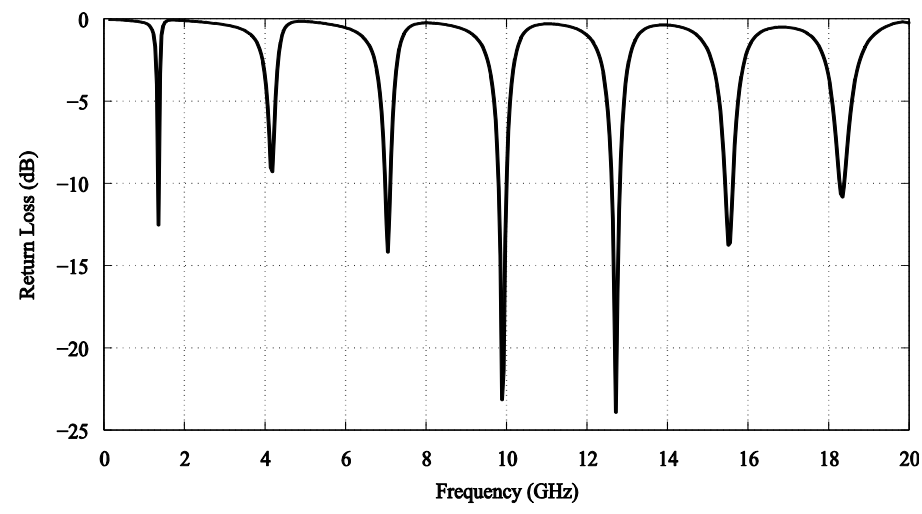

Fig. 2. Simulated input return loss of proposed multiband microstrip patch antenna.

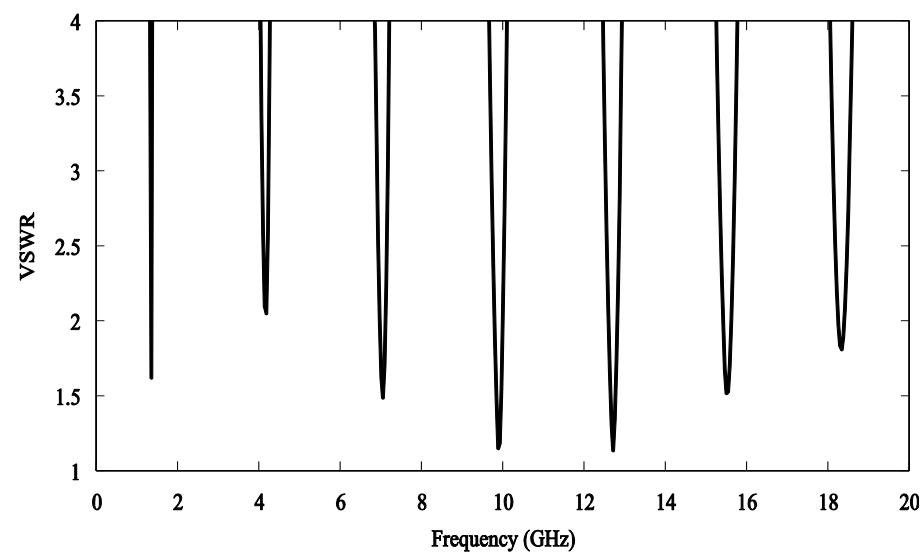

Fig. 3. Simulated VSWR of proposed multiband microstrip patch antenna. 


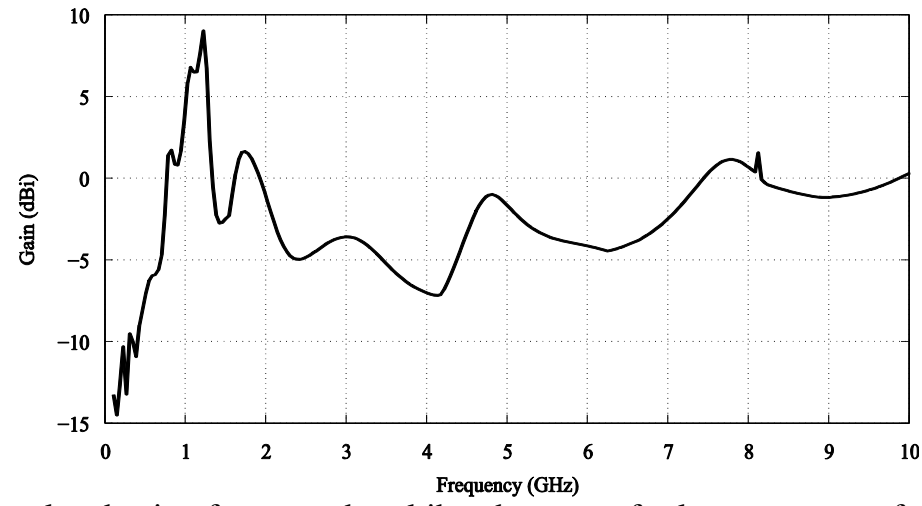

Fig. 4. Simulated gain of proposed multiband antenna for lower resonant frequencies.

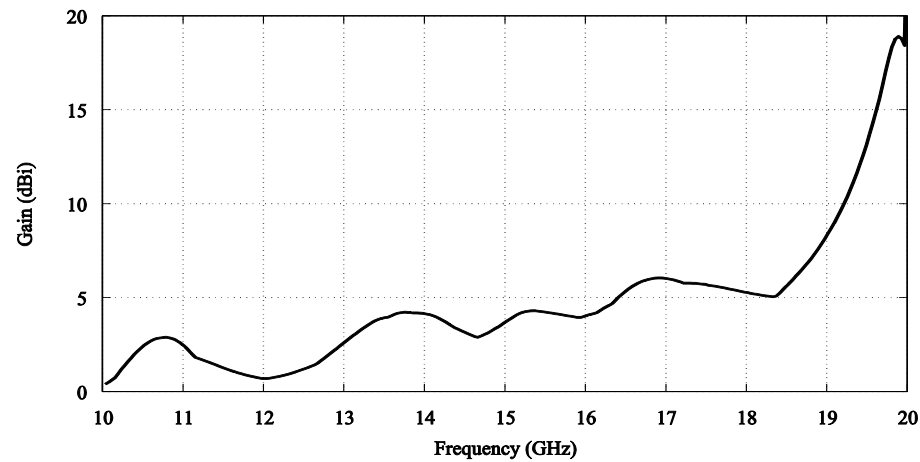

Fig. 5. Simulated gain of proposed multiband antenna for upper resonant frequencies.

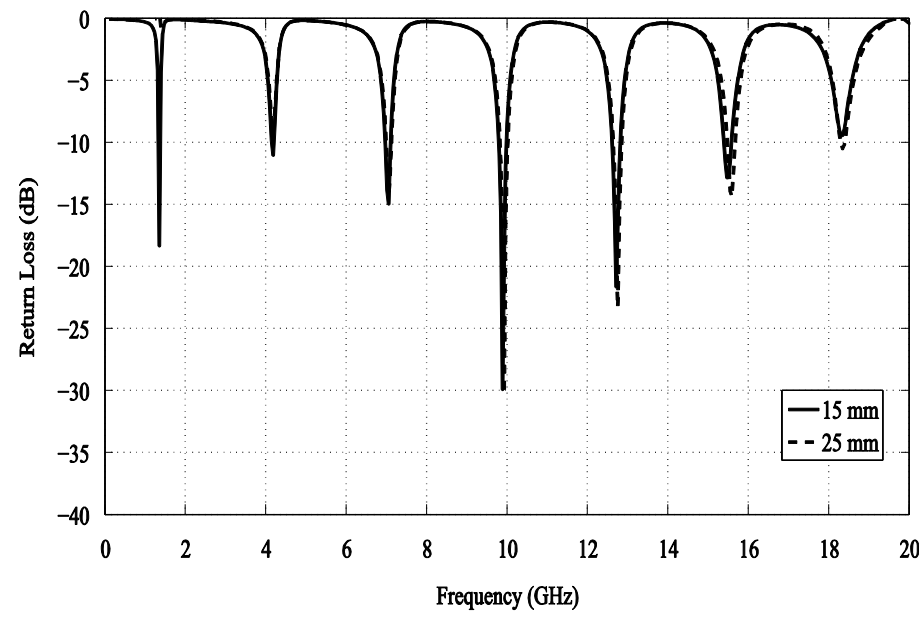

Fig. 6. Simulated input return loss by changing slot length $l_{l}$.

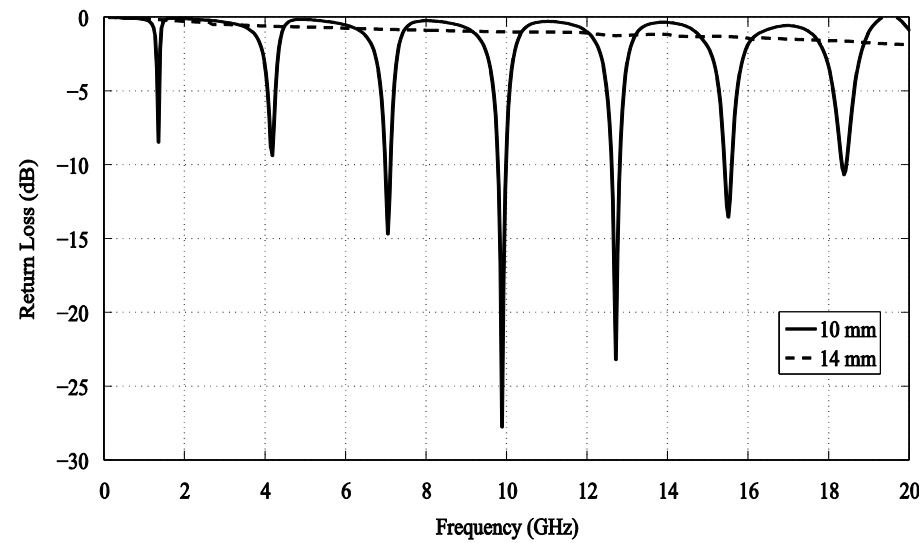

Fig. 7. Simulated input return loss by changing slot length $l_{2}$. 


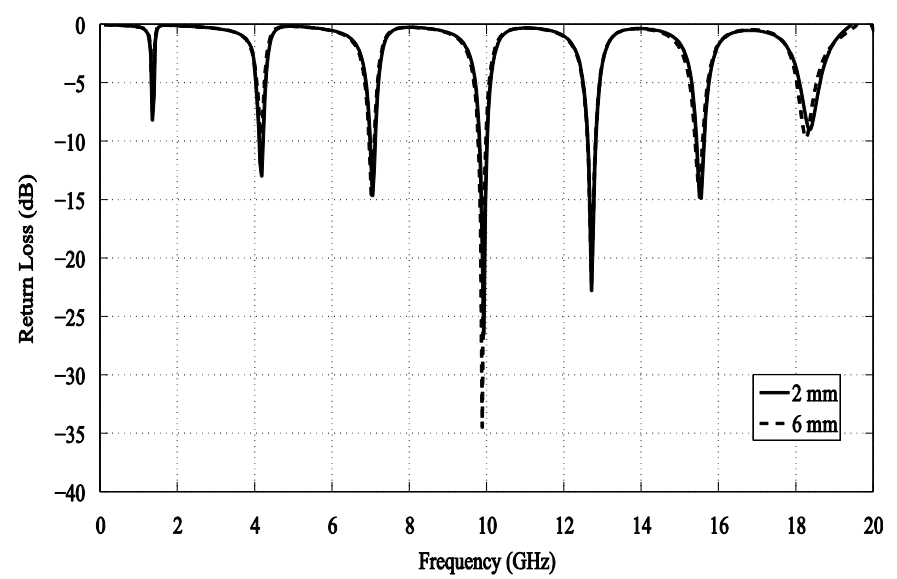

Fig. 8. Simulated input return loss by changing slot length $l_{3}$.

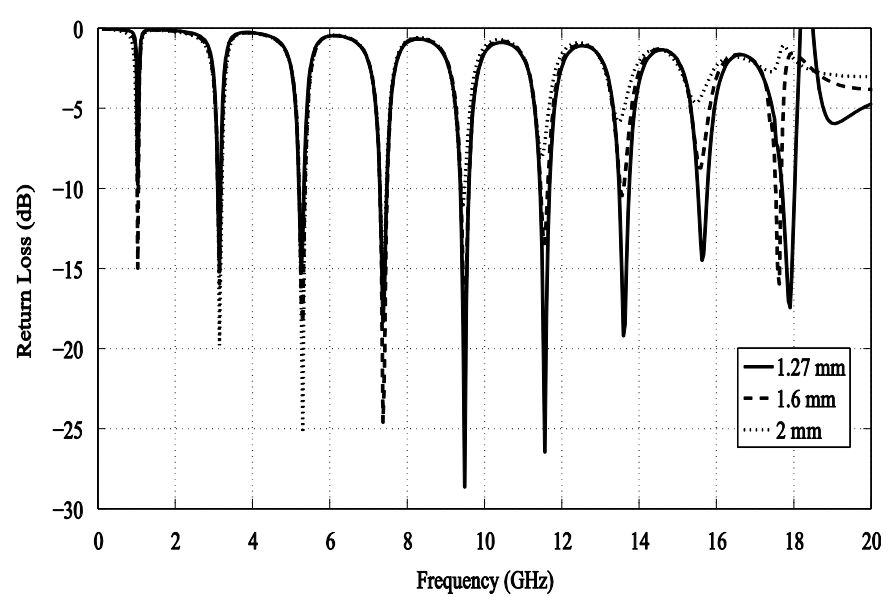

Fig. 9. Simulated input return loss by changing substrate/dielectric thickness $(h)$.

\section{References}

[1] C. A. Balanis, Antenna Theory and Design, $3^{\text {rd }}$ Edition.

[2] V.-A. Nguyel, R.-A. Bhatti and S.-O. Park, "A simple PIFA-based tunable internal antenna for personal communication handsets," IEEE Antennas Wireless Propag. Lett., vol. 7, pp. 130-133, 2008.

[3] N. Behbad and K. Sarabandi, "Dual-band reconfigurable antenna with a very wide tenability range," IEEE Trans. Antennas Propag., vol. 54, no. 2, pp. 409-416, 2006.

[4] H. Okabe and K. Takei, "Tunable antenna system for $1.9 \mathrm{GHz}$ PCS handsets," IEEE Antennas Propag. Int. Symp., vol. 1, pp. 166169, 2001.

[5] D. Peroulis, K. Sarabandi and L. B. P. Katehi, "Design of reconfigurable slot antennas," IEEE Trans. Antennas Propag., vol. 53, no. 7, pp. 645-654, 2005.

[6] F. Yang and Y. R. Samii, "A reconfigurable patch antenna using switchable slots for circular polarization diversity," IEEE Micro. Wireless Comp. Lett., vol. 12, no. 3, pp. 96-98, 2002.

[7] K.-M. Lee, Y.-J. Sung, J.-W. Baik and Y.-S. Kim, “A triangular microstrip patch antenna for multi-band applications,” Asia Pacific Microwave Conf., pp. 1-4, 2008.

[8] K. Chung, Y. Nam, T. Yun and J. Choi, "Reconfigurable microstrip patch antenna with switchable polarization," ETRI Journal, vol. 28, no. 3, 2006.

[9] P. Muthili, P. Cherian, S. Mridula and D. Paul, "Design of a compact multi-band microstrip antenna," 2009 Annual India Conf., pp. $1-4,2009$.

[10] G. Jegan, A. V. Juliet and G. A. Kumar, "Multiband microstrip patch antenna for satellite communication," Recent Adv. Space Tech. Services and Climate Change, pp. 153-156, 2010

[11] S. S. Patel and Y. P. Kosta, "Multiband PBG suspended patch antenna," $3^{\text {rd }}$ Int. Conf. Electronic and Computer Tech., vol. 2, pp. 59, 2011.

[12] H.-C. Ryu, H.-R. ahn, S.-H. Lee and W. S. Park, “Triple-stacked microstrip patch antenna for multiband system,” Elect. Lett., vol. 38, no. 24, pp. 1496-1497, 2002. 